\title{
Potential influencing factors on the outcome in incisional hernia repair: a registry-based multivariable analysis of 22,895 patients
}

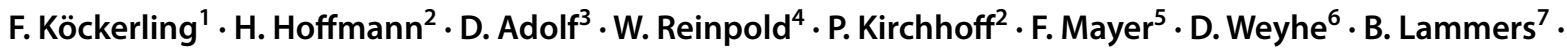 \\ K. Emmanuel ${ }^{5}$
}

Received: 4 February 2020 / Accepted: 30 March 2020 / Published online: 10 April 2020

(c) The Author(s) 2020

\begin{abstract}
Introduction Due to the paucity of randomized controlled trials, meta-analyses of incisional hernia repair can hardly give any insights into the influence factors on the various outcome criteria. Therefore, a multivariable analysis of data from the Herniamed Registry was undertaken with the aim to define potential influencing factors for the outcome.

Methods Multivariable analysis of the data available for 22,895 patients with primary elective incisional hernia repair was performed to assess the confirmatory predefined potential influence factors and their association with the perioperative and 1-year follow-up outcomes. A model validation procedure was implemented using a bootstrap algorithm in order to account for the robustness of results.

Results Higher European Hernia Society (EHS) width classification, open procedure, female gender, and preoperative pain have a highly significant association with an unfavorable outcome in incisional hernia repair. Larger defect width and open operation have a highly significantly unfavorable relation to the postoperative surgical complications, general complications, and the complication-related reoperations, while female gender and preoperative pain have a highly significantly unfavorable association with the rates of pain at rest, pain on exertion, and chronic pain requiring treatment at 1-year follow-up. The recurrence rate is significantly unfavorably influenced by higher EHS width classification, higher BMI, and lateral EHS classification.

Conclusion Higher EHS width classification, open procedure, female gender, higher BMI, and lateral EHS classification, as well as preoperative pain are the most important unfavorable influencing factors associated with a worse outcome in incisional hernia repair.
\end{abstract}

Keywords Incisional hernia $\cdot$ Outcome $\cdot$ EHS-classification $\cdot$ Laparoscopic IPOM $\cdot$ Sublay $\cdot$ Chronic pain

F. Köckerling

ferdinand.koeckerling@vivantes.de

1 Department of Surgery and Center for Minimally Invasive Surgery, Academic Teaching Hospital of Charité Medical School, Vivantes Hospital, Neue Bergstrasse 6, 13585 Berlin, Germany

2 Two Surgeons-Center for Hernia Surgery and Proctology, St. Johanns-Vorstadt 44, 4056 Basel, Switzerland

3 StatConsult GmbH, Halberstädter Strasse 40 a, 39112 Magdeburg, Germany

4 Department of Surgery, Wilhelmsburger Hospital Gross Sand, Academic Teaching Hospital of University Hamburg, Gross Sand 3, 21107 Hamburg, Germany
5 Department of Surgery, Paracelsus Medical University Salzburg, Müllner Hauptstrasse 48, 5020 Salzburg, Austria

6 Department of General and Visceral Surgery, Pius Hospital, University Hospital of Visceral Surgery, Georgstrasse 12, 26121 Oldenburg, Germany

7 Department of Surgery I-Section Coloproctology and Hernia Surgery, Lukas Hospital, Preussenstrasse 84, 41464 Neuss, Germany 


\section{Introduction}

Compared with primary ventral hernia repair, incisional ventral hernia repair has a significantly poorer perioperative and long-term outcomes [1-6]. Therefore, data on primary and incisional ventral hernias should not be jointly evaluated in studies [1-6]. Hence, meta-analysis findings that make no distinction between primary and incisional ventral hernia repair should be interpreted with caution [7]. The two meta-analyses focusing exclusively on incisional hernia were able to evaluate only a maximum of six randomized controlled trials with a total of 751 patients [8-11]. That small sample size with a relatively large number of factors potentially influencing the outcome is hardly suitable for reliable identification of the relevance of the various influence factors on the outcome in incisional hernia repair. The meta-analysis was only able to demonstrate that the wound complication rate in laparoscopic intraperitoneal onlay mesh (IPOM) was lower than in the open procedures [8-11]. There was no significant difference in the recurrence rates [8-11].

Registry and database analyses reported in the literature make several references to factors that have an unfavorable influence on the outcome following incisional hernia repair [12-16]. These unfavorable factors, include age, gender, risk factors, open surgical procedures, defect width, body mass index $(\mathrm{BMI}) \geq 30 \mathrm{~kg} / \mathrm{m}^{2}$ ), and smoking [12-16]. Recently, an analysis of the data of 2191 patients from the French Hernia Registry "Club Hernie" showed that larger defect widths, as classified by the European Hernia Society (EHS) [17], had an unfavorable impact on the postoperative complication rate [15].

This present analysis of data from the Herniamed Hernia Registry $[18,19]$ aims to assess potential influencing factors associated with outcome in incisional hernia repair. In particular, it seeks to evaluate the importance of the European Hernia Society width classification [17] as an unfavorable factor for the outcome in incisional hernia repair. To do so, the impact of confirmatory chosen, potential influencing factors on several outcome parameters was assessed, accounting for notable odds ratios and robust results.

\section{Materials and methods}

"The Herniamed quality assurance study is a multicenter internet-based hernia registry with voluntary participating institutions which incorporate prospective data of patients who have undergone routine hernia surgery" $[20,21]$.

"These data are obtained from 712 voluntarily participating hospitals and surgeons in Germany, Austria, and Switzerland" [20, 21]. All patients gave informed consent agreeing to participate [20,21]. "As part of the informed consent declaration, information provided to patients regarding participation in the Herniamed Registry included the request that the hospital or medical practice providing treatment would like to be informed about any problem occurring after the operation and that patients have the opportunity to attend clinical examination" [20, 21].

"At 1-year follow-up, postoperative complications are once again reviewed when the general practitioner and patient are asked to report any occurrences, pain at rest, pain on exertion, and chronic pain requiring treatment" $[20,21]$. "If recurrence or chronic pain is reported by the patient or the general practitioner the patients can be requested to present themselves for clinical or radiological examination" [20, 21]. A publication by Baucom et al. [22] has provided impressive evidence of the role of patientreported outcomes for both recurrence and chronic pain following incisional hernia repair.

In the current analysis, prospective data of patients who underwent primary elective incisional hernia repair with the laparoscopic intraperitoneal onlay mesh (IPOM) technique or open suture, sublay, onlay, or IPOM approach were evaluated to assess all confirmatory pre-defined potential influencing factors on the perioperative and 1-year follow-up outcomes. Here, the focus in particular was to assess the role of EHS width classification W1 $(<4 \mathrm{~cm}), \mathrm{W} 2(\geq 4 \mathrm{~cm}-10 \mathrm{~cm}), \mathrm{W} 3(>10 \mathrm{~cm})$ on the outcome [16]. Further variables to be assessed were age in years, BMI in $\mathrm{kg} / \mathrm{m}^{2}$, gender, ASA score, surgical technique, preoperative pain (yes, no, unknown), drainage (yes, no), EHS classification (medial, lateral, combined), presence of risk factors (yes, no), and postoperative complications (yes, no) on analysis of pain at follow-up.

Risk factors were deemed to apply if at least one of the following risk factors was present: COPD, diabetes mellitus, aortic aneurysm, immunosuppression, corticoids, smoking, coagulopathy, antiplatelet medication not adequately discontinued, or coumarin derivatives with Quick/ INR not in normal range.

The main inclusion criteria for the analysis population were minimum valid age of 16 years, primary elective incisional hernia repair using the laparoscopic IPOM or open suture, sublay, onlay, IPOM technique, no use of a Physiomesh [20], and availability of data at 1-year followup (Fig. 1). 22,895 patients fulfilled these inclusion criteria (Fig. 1).

Physiomesh was excluded from this analysis because of the voluntary market withdrawal [20]. Recurrent incisional hernias were also excluded for homogeneity of the patient population. 


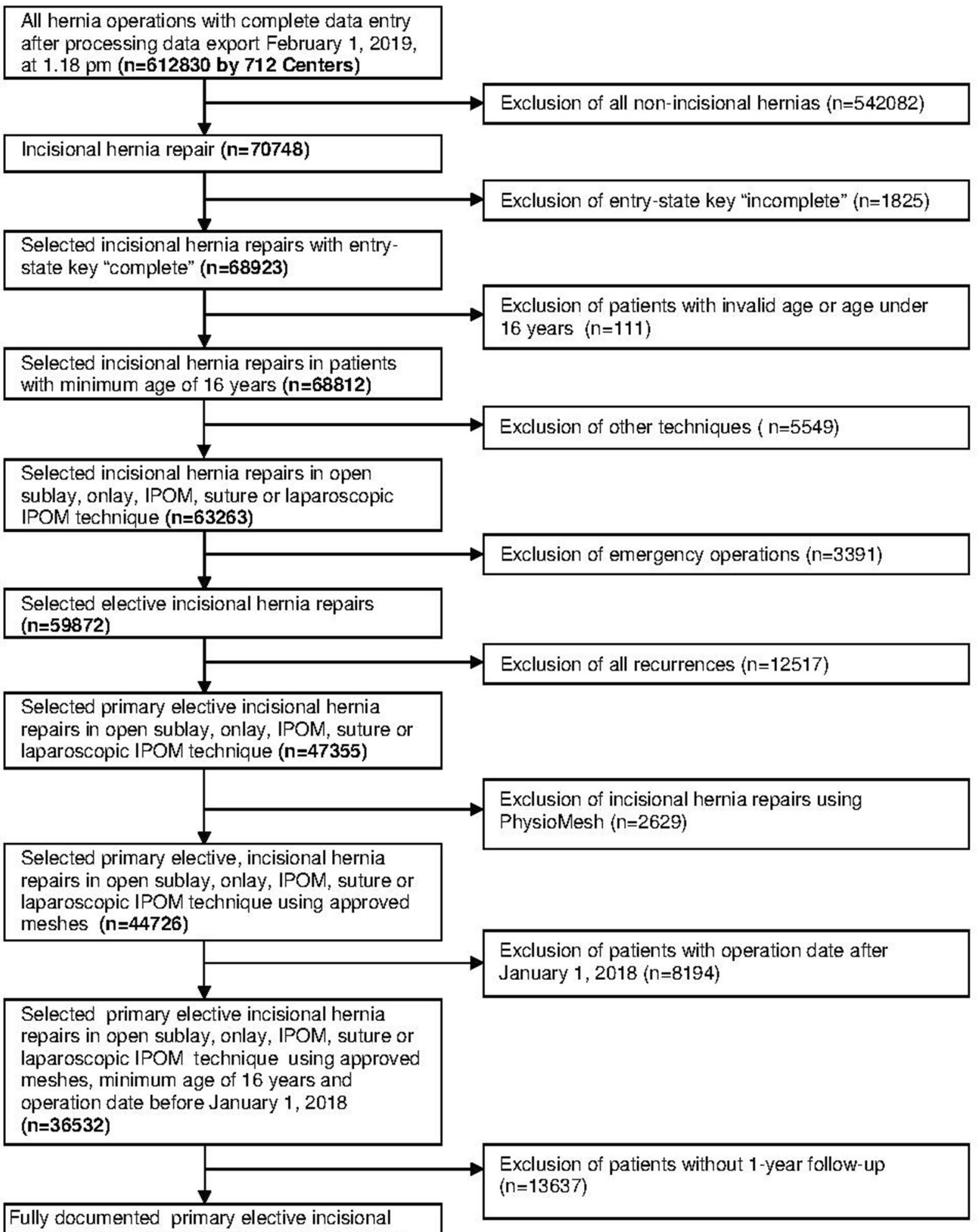

Fully documented prinary elective incisional hernia repairs using approved meshes in open sublay, onlay, IPOM, suture or laparoscopic IPOM technique, before January 1, 2018 with minimum age of 16 years and 1 -year follow-up $(n=22895)$

Fig. 1 Flowchart pf patient inclusion 
In total, 22,895 patients were selected between September 1, 2009 and January 1, 2018. Of these patients, 6361 $(27.8 \%)$ had undergone laparoscopic IPOM, 2662 (11.6\%) open suture, 9378 (41.0\%) open sublay, 3196 (13.9\%) open IPOM, and $1298(5.7 \%)$ open onlay procedure.

All analyses were performed with the software SAS 9.4 (SAS Institute Inc. Cary, NC, USA) and deliberately reviewed to the full level of significance. Each $p$ value $\leq 0.05$ thus represents a statistically significant result. Categorical variables are given as absolute and relative frequencies. For continuous data, mean and standard deviation or range of dispersion for log-transformed data, respectively, are given.

For unadjusted analyses of EHS width classification, the Chi-square test was used for categorical variables and an analysis of variance (ANOVA) was used for continuous variables. Analyses of non-normal distributed data (operating time and mesh size) were done on log-transformed values.

The potential influence of EHS width classification on the outcome parameters (intraoperative, postoperative and general complications, complication-related reoperations, as well as recurrence, pain at rest, pain on exertion, and pain requiring treatment at 1-year follow-up) adjusted for pre-defined confounding patient- and operation-related variables was analyzed via multivariable binary logistic models. Estimates for odds ratio (OR) and the corresponding $95 \%$ confidence interval are given. For independent variables with more than two categories, all pairwise odds ratios are provided. For the continuous variable "age" the 10 -year odds ratio, and for the variable "BMI" a five-point odds ratio, is given.

All available data were included in the models. Detailed results of outcome variables presented in this paper refer to the corresponding estimates in those single models. The robustness of the results in terms of stable odds ratio estimates was assessed using a bootstrap algorithm per model with 1000 bootstrap samples each.

Since significance can also be reached for very small effects in such a huge registry study, in order to facilitate evaluation of the relevance of individual influence variables on the various outcome criteria, and to account for robustness of results, the following definition of influence strength is applied in the summary presentation of results (Fig. 2):

- An odds ratio of $\geq 1.5$ with a corresponding $p$ value of $<0.001$ and consistent results in at least 3/4 of all bootstrap samples are defined as highly significantly unfavorable relation.

- An odds ratio of $<0.667$ with a corresponding $p$ value of $<0.001$ and consistent results in at least $3 / 4$ of all bootstrap samples refer to a highly significantly favorable relation.

\begin{tabular}{|c|c|c|c|c|c|c|c|c|c|c|c|}
\hline & $\begin{array}{l}\text { Higher EHS } \\
\text { Width } \\
\text { Classification } \\
\text { W3 vs w1 }\end{array}$ & $\begin{array}{l}\text { Higher EHS } \\
\text { Width } \\
\text { Classification } \\
\text { w2 vs w1 }\end{array}$ & $\begin{array}{l}\text { Open sublay } \\
\text { vs } \\
\text { laparoscopic } \\
\text { IPOM }\end{array}$ & Higher age & $\begin{array}{l}\text { Female } \\
\text { gender }\end{array}$ & $\begin{array}{l}\text { Risk factors } \\
\text { yes vs no }\end{array}$ & $\begin{array}{l}\text { Higher ASA } \\
\text { score } \\
\mid \| / / \text { v vs I }\end{array}$ & Higher BMI & $\begin{array}{l}\text { EHS } \\
\text { Classification } \\
\text { lateral vs } \\
\text { medial }\end{array}$ & $\begin{array}{l}\text { Preoperative } \\
\text { pain } \\
\text { yes vs no }\end{array}$ & $\begin{array}{l}\text { Postoperative } \\
\text { complications } \\
\text { yes vs no }\end{array}$ \\
\hline $\begin{array}{l}\text { Intraoperative } \\
\text { complication }\end{array}$ & -- & -- & ++ & - & 0 & 0 & 0 & 0 & 0 & 0 & \\
\hline $\begin{array}{l}\text { Postoperative } \\
\text { complication }\end{array}$ & -- & -- & -- & 0 & 0 & - & 0 & - & + & 0 & \\
\hline $\begin{array}{l}\text { Complication } \\
\text {-related } \\
\text { reoperation }\end{array}$ & -- & - & -- & 0 & 0 & - & 0 & 0 & + & 0 & \\
\hline $\begin{array}{l}\text { General } \\
\text { complication }\end{array}$ & -- & -- & 0 & - & o & - & - & 0 & + & 0 & \\
\hline Recurrence & - & - & + & 0 & 0 & 0 & 0 & - & - & 0 & \\
\hline Pain in rest & - & 0 & 0 & + & - & 0 & - & + & - & -- & -- \\
\hline $\begin{array}{l}\text { Pain on } \\
\text { exertion }\end{array}$ & - & - & 0 & + & -- & - & 0 & + & - & -- & - \\
\hline $\begin{array}{l}\text { Chronic pain } \\
\text { requiring } \\
\text { treatment }\end{array}$ & - & o & 0 & + & -- & - & -- & + & - & -- & - \\
\hline
\end{tabular}
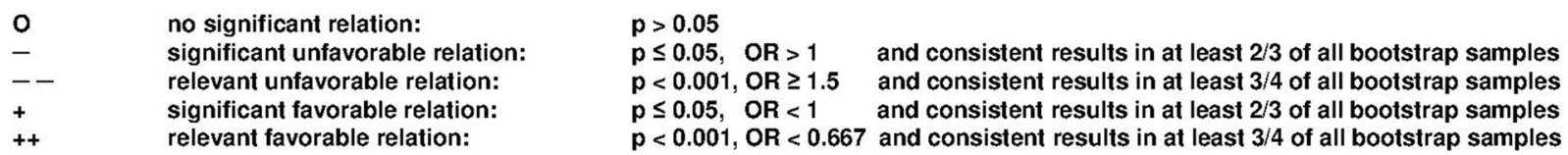

Fig. 2 Scheme of relationship between outcomes and potential influencing factors including information from bootstrap algorithm (not all pairwise comparisons shown) 
- A significant effect $(p \leq 0.05)$ with an odds ratio of $>1$ and consistent or even strengthened results in at least $2 / 3$ of all bootstrap samples indicate a significantly unfavorable relation

- A significant effect $(p \leq 0.05)$ with an odds ratio of $<1$ and consistent or even strengthened results in at least $2 / 3$ of all bootstrap samples define a significantly favorable relation.

\section{Results}

\section{Unadjusted analysis}

This section investigated deviations in the frequency distribution of the influence and outcome variables in relation to EHS width classification unadjusted for other potential influencing factors. The frequency distribution for EHS width classification revealed for $\mathrm{W} 1(<4 \mathrm{~cm}) n=8615$ cases (37.6\%), for $\mathrm{W} 2(\geq 4 \mathrm{~cm}-10 \mathrm{~cm}) n=10,519(45.9 \%)$ cases, and for W3 $(>10 \mathrm{~cm}) n=3761(16.4 \%)$ cases.

Patients with larger defects had a significantly higher age (Table 1). Likewise, the BMI $\left(\mathrm{kg} / \mathrm{m}^{2}\right)$ was significantly higher for larger defects. The mean operating time was notably longer for larger defects. As expected, the meshes used were on average significantly larger for higher EHS classification.

Although the proportion of male patients with increasing EHS width classification rose, the corresponding proportion of women declined (Table 2). Significant differences were also seen in the distribution of the operative techniques used with regard to EHS width classification. Likewise, a greater proportion of patients with higher width classification also showed higher ASA scores. The proportion of lateral EHS classifications declined in line with rising EHS width classification, whereas the proportion of combined EHS classifications increased significantly. The proportion of patients with preoperative pain declined significantly in line with increasing defect width. Patients with larger defect widths also had significantly more risk factors. Similarly, for increasing defect widths, surgeons used drains significantly more often.

Unadjusted analysis of the relationship between width classification and the intra- and postoperative surgical complications, general complications and complication-related reoperations, recurrences, as well as pain at rest, and on exertion and chronic pain requiring treatment at 1-year follow-up is presented in detail in Table 3. A significant relationship was identified between width classification and all outcome variables unadjusted for other potential influences. For all outcome parameters, the corresponding rate rose to a relevant degree in line with increasing width classification.

\section{Multivariable analysis}

\section{Intraoperative complications}

The risk of intraoperative complications (model fit: $p<0.001)$ was significantly related with the surgical technique, width classification, and the use of a drain (in each case $p<0.001)$, as well as age $(p=0.012)$ and gen$\operatorname{der}(p=0.024)$ (Table 4, Fig. 2). The open techniques were associated with a lower intraoperative complication risk. Furthermore, the use of drains and higher EHS width classifications were associated with higher intraoperative complication risk. Likewise, in older patients, there was a higher risk of intraoperative complications. Those estimated effects for EHS width classification correspond to differences of, e.g., 21 intraoperative complications for every 1000 operations with $\mathrm{W} 3$ as compared to nine intraoperative complications for every 1000 operations with W1 width classification.

\section{Postoperative complications}

The analysis results for postoperative complications are presented in Table 5 (model fit: $p<0.001$ ). The onset of postoperative complications was highly significantly associated with width classification, operative technique, BMI, presence of risk factors, use of a drain, and EHS classification (in each case $p<0.001$ ) and significantly with ASA score $(p=0.002)$, and age $(p=0.041)$ (Fig. 2). The wider

Table 1 Presentation of ranges and of unadjusted analysis results for homogeneity between width classification and age, BMI, operating time, and mesh size

\begin{tabular}{|c|c|c|c|c|c|}
\hline & & \multicolumn{3}{|l|}{ EHS width classification } & \multirow[t]{2}{*}{$p$} \\
\hline & & $\mathrm{W} 1(<4 \mathrm{~cm})$ & $\mathrm{W} 2(\geq 4-10 \mathrm{~cm})$ & $\mathrm{W} 3(>10 \mathrm{~cm})$ & \\
\hline Age [years] & $N /$ mean $\pm \mathrm{SD}$ & $8615 / 60.4 \pm 14.6$ & $10,519 / 64.4 \pm 12.5$ & $3761 / 64.9 \pm 11.9$ & $<0.001$ \\
\hline BMI $\left[\mathrm{kg} / \mathrm{m}^{2}\right]$ & $N /$ mean $\pm \mathrm{SD}$ & $8587 / 28.4 \pm 5.6$ & $10,474 / 29.5 \pm 5.8$ & $3747 / 29.9 \pm 5.9$ & $<0.001$ \\
\hline OP-time [min] $]^{\mathrm{a}}$ & $N /$ mean [range] & $7978 / 50.4[48.7 ; 52.1]$ & $10,492 / 80.5[78.9 ; 82.2]$ & $3759 / 110.8[109.2 ; 112.3]$ & $<0.001$ \\
\hline Mesh size $\left[\mathrm{cm}^{2}\right]^{\mathrm{a}}$ & $N /$ mean [range] & $6274 / 118.4[116.0 ; 120.9]$ & $9953 / 270.1[268.2 ; 272.1]$ & $3643 / 487.2[485.5 ; 488.8]$ & $<0.001$ \\
\hline
\end{tabular}

${ }^{a}$ Logarithmic transformation: illustration of the back-transformed mean values and ranges (mean value \pm SD) 
Table 2 Presentation of descriptive statistics and of unadjusted analysis results for homogeneity between width classification and categorical influencing variables

\begin{tabular}{|c|c|c|c|c|c|c|c|}
\hline & \multicolumn{6}{|c|}{ EHS width classification } & \multirow[t]{3}{*}{$p$} \\
\hline & \multicolumn{2}{|c|}{$\mathrm{W} 1(<4 \mathrm{~cm})$} & \multicolumn{2}{|c|}{$\mathrm{W} 2(\geq 4-10 \mathrm{~cm})$} & \multicolumn{2}{|c|}{$\mathrm{W} 3(>10 \mathrm{~cm})$} & \\
\hline & $n$ & $\%$ & $n$ & $\%$ & $n$ & $\%$ & \\
\hline \multicolumn{8}{|l|}{ Gender } \\
\hline Male & 4132 & 47.96 & 5245 & 49.86 & 2038 & 54.19 & $<0.001$ \\
\hline Female & 4483 & 52.04 & 5274 & 50.14 & 1723 & 45.81 & \\
\hline \multicolumn{8}{|l|}{ Procedure } \\
\hline Laparoscopic_-IPOM & 2469 & 28.66 & 3099 & 29.46 & 793 & 21.08 & $<0.001$ \\
\hline Open-suture & 2223 & 25.80 & 377 & 3.58 & 62 & 1.65 & \\
\hline Open-IPOM & 1219 & 14.15 & 1283 & 12.20 & 694 & 18.45 & \\
\hline Open-onlay & 440 & 5.11 & 649 & 6.17 & 209 & 5.56 & \\
\hline Open-sublay & 2264 & 26.28 & 5111 & 48.59 & 2003 & 53.26 & \\
\hline \multicolumn{8}{|l|}{ ASA score } \\
\hline I & 1460 & 16.95 & 901 & 8.57 & 242 & 6.43 & $<0.001$ \\
\hline II & 5047 & 58.58 & 5952 & 56.58 & 1971 & 52.41 & \\
\hline III/IV & 2108 & 24.47 & 3666 & 34.85 & 1548 & 41.16 & \\
\hline \multicolumn{8}{|l|}{ EHS classification } \\
\hline Combined & 495 & 5.75 & 846 & 8.04 & 537 & 14.28 & $<0.001$ \\
\hline Lateral & 1716 & 19.92 & 1823 & 17.33 & 428 & 11.38 & \\
\hline Medial & 6404 & 74.34 & 7850 & 74.63 & 2796 & 74.34 & \\
\hline \multicolumn{8}{|l|}{ Preoperative pain } \\
\hline Yes & 5178 & 60.10 & 5842 & 55.54 & 2068 & 54.99 & $<0.001$ \\
\hline No & 2741 & 31.82 & 3745 & 35.60 & 1373 & 36.51 & \\
\hline Unknown & 696 & 8.08 & 932 & 8.86 & 320 & 8.51 & \\
\hline \multicolumn{8}{|l|}{ Drainage } \\
\hline Yes & 2893 & 33.58 & 6748 & 64.15 & 2843 & 75.59 & $<0.001$ \\
\hline No & 5722 & 66.42 & 3771 & 35.85 & 918 & 24.41 & \\
\hline \multicolumn{8}{|l|}{ Risk factors } \\
\hline \multicolumn{8}{|l|}{ Total } \\
\hline Yes & 3091 & 35.88 & 4497 & 42.75 & 1794 & 47.70 & $<0.001$ \\
\hline No & 5524 & 64.12 & 6022 & 57.25 & 1967 & 52.30 & \\
\hline \multicolumn{8}{|l|}{ COPD } \\
\hline Yes & 755 & 8.76 & 1134 & 10.78 & 457 & 12.15 & $<0.001$ \\
\hline No & 7860 & 91.24 & 9385 & 89.22 & 3304 & 87.85 & \\
\hline \multicolumn{8}{|l|}{ Diabetes } \\
\hline Yes & 884 & 10.26 & 1482 & 14.09 & 626 & 16.64 & $<0.001$ \\
\hline No & 7731 & 89.74 & 9037 & 85.91 & 3135 & 83.36 & \\
\hline \multicolumn{8}{|l|}{ Aortic aneurysm } \\
\hline Yes & 67 & 0.78 & 196 & 1.86 & 107 & 2.84 & $<0.001$ \\
\hline No & 8548 & 99.22 & 10,323 & 98.14 & 3654 & 97.16 & \\
\hline \multicolumn{8}{|l|}{ Immunosuppression } \\
\hline Yes & 109 & 1.27 & 202 & 1.92 & 95 & 2.53 & $<0.001$ \\
\hline No & 8506 & 98.73 & 10,317 & 98.08 & 3666 & 97.47 & \\
\hline \multicolumn{8}{|l|}{ Corticoid } \\
\hline Yes & 124 & 1.44 & 179 & 1.70 & 86 & 2.29 & 0.004 \\
\hline No & 8491 & 98.56 & 10,340 & 98.30 & 3675 & 97.71 & \\
\hline \multicolumn{8}{|l|}{ Smoking } \\
\hline Yes & 1007 & 11.69 & 1225 & 11.65 & 515 & 13.69 & 0.002 \\
\hline No & 7608 & 88.31 & 9294 & 88.35 & 3246 & 86.31 & \\
\hline
\end{tabular}


Table 2 (continued)

\begin{tabular}{|c|c|c|c|c|c|c|c|}
\hline & \multicolumn{6}{|c|}{ EHS width classification } & \multirow[t]{3}{*}{$p$} \\
\hline & \multicolumn{2}{|c|}{$\mathrm{W} 1(<4 \mathrm{~cm})$} & \multicolumn{2}{|c|}{$\mathrm{W} 2(\geq 4-10 \mathrm{~cm})$} & \multicolumn{2}{|c|}{$\mathrm{W} 3(>10 \mathrm{~cm})$} & \\
\hline & $n$ & $\%$ & $n$ & $\%$ & $n$ & $\%$ & \\
\hline \multicolumn{8}{|c|}{ Coagulopathy } \\
\hline Yes & 137 & 1.59 & 243 & 2.31 & 95 & 2.53 & $<0.001$ \\
\hline No & 8478 & 98.41 & 10,276 & 97.69 & 3666 & 97.47 & \\
\hline \multicolumn{8}{|c|}{ ASS/plavix antiplatelet medication } \\
\hline Yes & 841 & 9.76 & 1353 & 12.86 & 535 & 14.22 & $<0.001$ \\
\hline No & 7774 & 90.24 & 9166 & 87.14 & 3226 & 85.78 & \\
\hline \multicolumn{8}{|c|}{ Anticoagulation therapy } \\
\hline Yes & 248 & 2.88 & 341 & 3.24 & 123 & 3.27 & 0.293 \\
\hline No & 8367 & 97.12 & 10,178 & 96.76 & 3638 & 96.73 & \\
\hline
\end{tabular}

Table 3 Presentation of descriptive statistics and unadjusted analysis results for homogeneity between width classification and outcome variables

\begin{tabular}{|c|c|c|c|c|c|c|c|}
\hline & \multicolumn{6}{|c|}{ EHS width classification } & \multirow[t]{3}{*}{$p$} \\
\hline & \multicolumn{2}{|c|}{$\mathrm{W} 1(<4 \mathrm{~cm})$} & \multicolumn{2}{|c|}{$\mathrm{W} 2(\geq 4-10 \mathrm{~cm})$} & \multicolumn{2}{|c|}{$\mathrm{W} 3(>10 \mathrm{~cm})$} & \\
\hline & $n$ & $\%$ & $N$ & $\%$ & $n$ & $\%$ & \\
\hline \multicolumn{8}{|c|}{ Intraoperative complication } \\
\hline Yes & 87 & 1.01 & 221 & 2.10 & 93 & 2.47 & $<0.001$ \\
\hline No & 8528 & 98.99 & 10,298 & 97.90 & 3668 & 97.53 & \\
\hline \multicolumn{8}{|c|}{ Postoperative complication } \\
\hline Yes & 353 & 4.10 & 863 & 8.20 & 516 & 13.72 & $<0.001$ \\
\hline No & 8262 & 95.90 & 9656 & 91.80 & 3245 & 86.28 & \\
\hline \multicolumn{8}{|c|}{ General complication } \\
\hline Yes & 161 & 1.87 & 398 & 3.78 & 232 & 6.17 & $<0.001$ \\
\hline No & 8454 & 98.13 & 10,121 & 96.22 & 3529 & 93.83 & \\
\hline \multicolumn{8}{|c|}{ Complication-related reoperation } \\
\hline Yes & 148 & 1.72 & 381 & 3.62 & 222 & 5.90 & $<0.001$ \\
\hline No & 8467 & 98.28 & 10,138 & 96.38 & 3539 & 94.10 & \\
\hline \multicolumn{8}{|c|}{ Recurrence on 1-year-follow-up } \\
\hline Yes & 377 & 4.38 & 528 & 5.02 & 206 & 5.48 & 0.018 \\
\hline No & 8238 & 95.62 & 9991 & 94.98 & 3555 & 94.52 & \\
\hline \multicolumn{8}{|c|}{ Pain on exertion on 1-year-follow-up } \\
\hline Yes & 1425 & 16.54 & 1948 & 18.52 & 763 & 20.29 & $<0.001$ \\
\hline No & 7190 & 83.46 & 8571 & 81.48 & 2998 & 79.71 & \\
\hline \multicolumn{8}{|c|}{ Pain in rest on 1-year-follow-up } \\
\hline Yes & 798 & 9.26 & 1041 & 9.90 & 418 & 11.11 & 0.006 \\
\hline No & 7817 & 90.74 & 9478 & 90.10 & 3343 & 88.89 & \\
\hline \multicolumn{8}{|c|}{ Pain requiring treatment on 1-year-follow-up } \\
\hline Yes & 591 & 6.86 & 806 & 7.66 & 320 & 8.51 & 0.004 \\
\hline No & 8024 & 93.14 & 9713 & 92.34 & 3441 & 91.49 & \\
\hline
\end{tabular}

the hernia, the higher the risk of postoperative complications, which results in, e.g., 99 postoperative complications for every 1,000 operations with W3 as compared to 42 for every $1000 \mathrm{~W} 1$ hernias. With regards to the operative technique, a reduction in the overall postoperative complication risk was achieved by using, in particular, the laparoscopic IPOM procedure. By comparison, the open procedures exhibited significantly higher-mostly twofold-risks. Higher BMI was associated with an increase in the postoperative complication rate. In contrast, lateral EHS classification, in particular in comparison with medial EHS classification, reduced the complication risk. 
Table 4 Multivariable analysis results for intraoperative complications, including odds ratio estimates with corresponding $95 \%$ confidence intervals

\begin{tabular}{|c|c|c|c|c|c|c|}
\hline Parameter & $p$ value & Category & $p$ value (pairwise) & OR estimate & $95 \% \mathrm{CI}$ & \\
\hline \multirow[t]{10}{*}{ Procedure } & \multirow[t]{10}{*}{$<0.001$} & Open—sublay vs laparoscopic_-IPOM & $<0.001$ & 0.303 & 0.226 & 0.405 \\
\hline & & Open—suture vs open—sublay & $<0.001$ & 3.169 & 2.206 & 4.552 \\
\hline & & Open-IPOM vs laparoscopic_-IPOM & $<0.001$ & 0.483 & 0.343 & 0.680 \\
\hline & & Open—onlay vs laparoscopic_-IPOM & $<0.001$ & 0.430 & 0.269 & 0.686 \\
\hline & & Open—suture vs open-IPOM & 0.001 & 1.987 & 1.319 & 2.992 \\
\hline & & Open—suture vs open—onlay & 0.002 & 2.232 & 1.339 & 3.723 \\
\hline & & Open-IPOM vs open—sublay & 0.004 & 1.595 & 1.157 & 2.198 \\
\hline & & Open—onlay vs open—sublay & 0.120 & 1.420 & 0.913 & 2.207 \\
\hline & & Open—onlay vs open-IPOM & 0.640 & 0.890 & 0.546 & 1.450 \\
\hline & & Open—suture vs laparoscopic-IPOM & 0.822 & 0.959 & 0.669 & 1.376 \\
\hline Drainage & $<0.001$ & Yes vs no & & 2.657 & 2.035 & 3.468 \\
\hline \multirow[t]{3}{*}{ EHS width classification } & \multirow[t]{3}{*}{$<0.001$} & $\mathrm{~W} 3(>10 \mathrm{~cm})$ vs W1 $(<4 \mathrm{~cm})$ & $<0.001$ & 2.399 & 1.730 & 3.326 \\
\hline & & $\mathrm{W} 2(\geq 4-10 \mathrm{~cm})$ vs W1 $(<4 \mathrm{~cm})$ & $<0.001$ & 2.036 & 1.548 & 2.678 \\
\hline & & $\mathrm{W} 3(>10 \mathrm{~cm})$ vs W2 $(\geq 4-10 \mathrm{~cm})$ & 0.198 & 1.178 & 0.918 & 1.512 \\
\hline Age [10-years-OR] & 0.012 & & & 1.117 & 1.024 & 1.218 \\
\hline Gender & 0.024 & Female vs male & & 1.266 & 1.032 & 1.553 \\
\hline \multirow[t]{3}{*}{ EHS classification } & \multirow[t]{3}{*}{0.272} & Lateral vs medial & 0.108 & 0.790 & 0.592 & 1.053 \\
\hline & & Lateral vs combined & 0.418 & 0.840 & 0.550 & 1.282 \\
\hline & & Combined vs medial & 0.730 & 0.941 & 0.664 & 1.333 \\
\hline \multirow[t]{3}{*}{ ASA score } & \multirow[t]{3}{*}{0.383} & II vs I & 0.183 & 0.787 & 0.554 & 1.120 \\
\hline & & III/IV vs I & 0.386 & 0.841 & 0.569 & 1.244 \\
\hline & & III/IV vs II & 0.570 & 1.068 & 0.851 & 1.341 \\
\hline BMI [5-points-OR] & 0.455 & & & 0.966 & 0.883 & 1.057 \\
\hline \multirow[t]{3}{*}{ Preoperative pain } & \multirow[t]{3}{*}{0.818} & Unknown vs no & 0.527 & 1.124 & 0.782 & 1.616 \\
\hline & & Yes vs unknown & 0.595 & 0.910 & 0.644 & 1.287 \\
\hline & & Yes vs no & 0.834 & 1.024 & 0.823 & 1.274 \\
\hline Risk factors & 0.840 & Yes vs no & & 0.978 & 0.791 & 1.211 \\
\hline
\end{tabular}

\section{Complication-related reoperations}

The analysis results for the complication-related reoperations (model fit; $p<0.001$ ) (Table 6, Fig. 2) showed that the risk of complication-related reoperation was significantly associated with hernia width, use of a drain, presence of risk factors, surgical technique (in each case $p<0.001$ ), EHS classification $(p=0.003)$, as well as ASA score, and BMI $(p=0.019)$. The complication-related reoperation risk-like the postoperative complication rates above-was notably related to higher width classification, resulting in, e.g., addition of 21 cases for every 1000 hernias with W3 (40/1000) as compared to W1 (19/1000). The use of drains, as well as the presence of at least one risk factor was also associated with a higher complication-related reoperation risk, whereas on comparing the operative techniques, in particular the use of a laparoscopic IPOM procedure reduced the risk. The latter also applied for lateral EHS classification, whereas higher ASA score and higher BMI were associated with a higher risk.

\section{General complications}

The general complications (model fit: $p<0.001$ ) were significantly related to width classification, presence of risk factors, age, use of a drain, and ASA score (in each case $p<0.001)$, as well as EHS classification $(p=0.003)$ (Table 7, Fig. 2). As in the case of the postoperative complications-also in the effect size for somewhat lower prevalence-wider hernias increased the risk of general complications between 48 and $140 \%$. The latter revealed 25 more cases with general complications for every 1,000 hernias with W3 (44/1000) as compared to W1 width. Independently of the above, risk factors, higher age and higher ASA score, and the use of a drain were associated with higher general complication risk. Conversely, lateral EHS classification showed a reduced complication risk. 
Table 5 Multivariable analysis results for postoperative complications, including odds ratio estimates with corresponding $95 \%$ confidence intervals

\begin{tabular}{|c|c|c|c|c|c|c|}
\hline Parameter & $p$ value & Category & $p$ value (pairwise) & OR estimate & $95 \% \mathrm{CI}$ & \\
\hline \multirow[t]{3}{*}{ EHS width classification } & $<0.001$ & $\mathrm{~W} 3(>10 \mathrm{~cm})$ vs W1 $(<4 \mathrm{~cm})$ & $<0.001$ & 2.500 & 2.135 & 2.927 \\
\hline & & W3 $(>10 \mathrm{~cm})$ vs W2 $(\geq 4-10 \mathrm{~cm})$ & $<0.001$ & 1.555 & 1.380 & 1.752 \\
\hline & & $\mathrm{W} 2(\geq 4-10 \mathrm{~cm})$ vs W1 $(<4 \mathrm{~cm})$ & $<0.001$ & 1.608 & 1.397 & 1.850 \\
\hline \multirow[t]{10}{*}{ Procedure } & $<0.001$ & Open—sublay vs laparoscopic_-IPOM & $<0.001$ & 2.291 & 1.917 & 2.738 \\
\hline & & Open—onlay vs laparoscopic_-IPOM & $<.001$ & 2.449 & 1.914 & 3.133 \\
\hline & & Open-IPOM vs laparoscopic_-IPOM & $<0.001$ & 1.977 & 1.622 & 2.410 \\
\hline & & Open-suture vs laparoscopic-IPOM & $<0.001$ & 1.739 & 1.362 & 2.220 \\
\hline & & Open—suture vs open—sublay & 0.013 & 0.759 & 0.611 & 0.943 \\
\hline & & Open—suture vs open—onlay & 0.014 & 0.710 & 0.540 & 0.934 \\
\hline & & Open-IPOM vs open—sublay & 0.051 & 0.863 & 0.744 & 1.001 \\
\hline & & Open—onlay vs open—IPOM & 0.063 & 1.239 & 0.988 & 1.552 \\
\hline & & Open—suture vs open-IPOM & 0.289 & 0.880 & 0.694 & 1.115 \\
\hline & & Open—onlay vs open—sublay & 0.504 & 1.069 & 0.879 & 1.300 \\
\hline BMI [5-points-OR] & $<0.001$ & & & 1.148 & 1.100 & 1.198 \\
\hline Drainage & $<0.001$ & Yes vs no & & 1.436 & 1.244 & 1.657 \\
\hline Risk factors & $<0.001$ & Yes vs no & & 1.293 & 1.163 & 1.437 \\
\hline \multirow[t]{3}{*}{ EHS classification } & $<0.001$ & Lateral vs medial & $<0.001$ & 0.727 & 0.625 & 0.844 \\
\hline & & Lateral vs combined & 0.066 & 0.812 & 0.650 & 1.014 \\
\hline & & Combined vs medial & 0.232 & 0.895 & 0.746 & 1.074 \\
\hline \multirow[t]{3}{*}{ ASA score } & 0.002 & III/IV vs II & $<0.001$ & 1.216 & 1.086 & 1.360 \\
\hline & & III/IV vs I & 0.030 & 1.273 & 1.023 & 1.583 \\
\hline & & II vs I & 0.656 & 1.047 & 0.856 & 1.280 \\
\hline Age [10-years-OR] & 0.041 & & & 1.047 & 1.002 & 1.094 \\
\hline Gender & 0.248 & Female vs male & & 0.941 & 0.850 & 1.043 \\
\hline \multirow[t]{3}{*}{ Preoperative pain } & 0.441 & Yes vs no & 0.204 & 1.074 & 0.962 & 1.198 \\
\hline & & Yes vs unknown & 0.662 & 1.042 & 0.866 & 1.255 \\
\hline & & Unknown vs no & 0.764 & 1.030 & 0.849 & 1.250 \\
\hline
\end{tabular}

\section{Recurrence}

The multivariable analysis results for analysis of recurrence at 1-year follow-up are given in Table 8 (model fit: $p<0.001)$. The recurrence was strongly associated with surgical technique, EHS width classification, EHS classification, and BMI (in each case $p<0.001$ ) (Fig. 2). The recurrence rate was increased, in particular, by the use of the open procedure with direct suture. Open sublay had a lower recurrence risk when compared with laparoscopic IPOM. Furthermore, larger defects, lateral EHS in comparison with medial and higher BMI were associated with higher recurrence risk. Here, the difference in recurrences is about 20 cases for every 1000 hernias with W3 (57/1000) as compared to W1 (37/1000).

\section{Pain at rest}

The analysis results for pain at rest at 1-year follow-up are summarized in Table 9 (model fit: $p<0.001$ ). This was highly and significantly associated with age, preoperative pain, gender, EHS classification, postoperative complications, and ASA score $(p<0.001)$, as well as with BMI $(p=0.003)$, operative technique $(p=0.027)$, use of a drain $(p=0.032)$, and also hernia width ( $p=0.033)$ Fig. 2$)$. Higher age and higher BMI led to less pain at rest. On the other hand, preoperative pain, lateral EHS, as well as combined classification compared with medial, postoperative complications, and the use of a drain showed an increased risk of pain at rest. Besides, women were at higher risk of pain at rest at 1-year follow-up than men. The association between operative technique and risk of pain at rest was reflected primarily in the reduced risk posed by the open suture technique. Finally, width classification was also found to exert a significant influence on pain at rest, but that significant impact was identified only on comparing W3 vs. W1 and the impact was of a lesser degree, with 106 out of 1000 patients with W3 classification suffering from pain at rest compared to 90 out of 1000 patients with W1. 
Table 6 Multivariable analysis results for complication-related reoperations, including odds ratio estimates with corresponding 95\% confidence intervals

\begin{tabular}{|c|c|c|c|c|c|c|}
\hline Parameter & $p$ value & Category & $p$ value (pairwise) & OR estimate & $95 \% \mathrm{KI}$ & \\
\hline \multirow[t]{3}{*}{ EHS width classification } & \multirow[t]{3}{*}{$<0.001$} & $\mathrm{~W} 3(>10 \mathrm{~cm})$ vs W1 $(<4 \mathrm{~cm})$ & $<0.001$ & 2.219 & 1.756 & 2.804 \\
\hline & & W3 $(>10 \mathrm{~cm})$ vs W2 $(\geq 4-10 \mathrm{~cm})$ & $<0.001$ & 1.438 & 1.209 & 1.711 \\
\hline & & $\mathrm{W} 2(\geq 4-10 \mathrm{~cm})$ vs W1 $(<4 \mathrm{~cm})$ & $<0.001$ & 1.543 & 1.251 & 1.903 \\
\hline Drainage & $<0.001$ & Yes vs no & & 1.851 & 1.480 & 2.315 \\
\hline Risk factors & $<0.001$ & Yes vs no & & 1.439 & 1.231 & 1.683 \\
\hline \multirow[t]{10}{*}{ Procedure } & \multirow[t]{10}{*}{$<0.001$} & Open—sublay vs laparoscopic-IPOM & $<0.001$ & 1.927 & 1.475 & 2.516 \\
\hline & & Open—onlay vs laparoscopic_-IPOM & $<0.001$ & 1.973 & 1.367 & 2.846 \\
\hline & & Open-IPOM vs laparoscopic_-IPOM & 0.002 & 1.594 & 1.180 & 2.155 \\
\hline & & Open—suture vs open—sublay & 0.037 & 0.698 & 0.497 & 0.979 \\
\hline & & Open—suture vs open—onlay & 0.073 & 0.681 & 0.448 & 1.036 \\
\hline & & Open-IPOM vs open-sublay & 0.094 & 0.827 & 0.663 & 1.033 \\
\hline & & Open—suture vs laparoscopic-IPOM & 0.127 & 1.344 & 0.919 & 1.966 \\
\hline & & Open—onlay vs open—IPOM & 0.214 & 1.237 & 0.884 & 1.731 \\
\hline & & Open-suture vs open-IPOM & 0.367 & 0.843 & 0.582 & 1.222 \\
\hline & & Open—onlay vs open—Sublay & 0.873 & 1.024 & 0.768 & 1.365 \\
\hline \multirow[t]{3}{*}{ EHS classification } & \multirow[t]{3}{*}{0.003} & Lateral vs medial & $<0.001$ & 0.675 & 0.535 & 0.851 \\
\hline & & Lateral vs combined & 0.012 & 0.659 & 0.476 & 0.911 \\
\hline & & Combined vs medial & 0.855 & 1.024 & 0.794 & 1.321 \\
\hline \multirow[t]{3}{*}{ ASA score } & \multirow[t]{3}{*}{0.016} & III/IV vs II & 0.004 & 1.273 & 1.079 & 1.502 \\
\hline & & III/IV vs I & 0.171 & 1.249 & 0.908 & 1.719 \\
\hline & & II vs I & 0.901 & 0.981 & 0.730 & 1.319 \\
\hline BMI [5-points-OR] & 0.019 & & & 1.079 & 1.013 & 1.149 \\
\hline Gender & 0.254 & Female vs male & & 0.916 & 0.788 & 1.065 \\
\hline \multirow[t]{3}{*}{ Preoperative pain } & \multirow[t]{3}{*}{0.313} & Yes vs no & 0.189 & 1.115 & 0.948 & 1.312 \\
\hline & & Unknown vs no & 0.227 & 1.186 & 0.899 & 1.563 \\
\hline & & Yes vs unknown & 0.648 & 0.941 & 0.723 & 1.224 \\
\hline Age [10-years-OR] & 0.836 & & & 0.993 & 0.932 & 1.059 \\
\hline
\end{tabular}

\section{Pain on exertion}

Pain on exertion at 1-year follow-up, (model fit: $p<0.001$ ), was highly significantly associated with age, gender, preoperative pain, EHS classification, postoperative complications, hernia width, operative technique, use of a drain (in each case $p<0.001)$, as well as BMI $(p=0.004)$ and presence of risk factors ( $p=0.004)$ (Fig. 2 and Table 10). Higher age and higher BMI indicate a reduced risk of pain on exertion. On the other hand, women had a notably higher risk of pain in comparison with men. Preoperative pain, lateral EHS and combined vs. medial, postoperative complications, higher width classification, and presence of at least one risk factor likewise increased the risk of pain on exertion. The use of drains was also associated with a higher risk of pain on exertion. Likewise, the operative technique was found to have a significant impact. Here, too, that was reflected in particular in the advantages conferred by the open suture procedure, as shown by the above estimates. The difference in cases of pain on exertion is about 44 for every 1000 hernias with W3 (199/1000) compared to W1 width classification (155/1000).

\section{Chronic pain requiring treatment}

The analysis results for pain requiring treatment are presented in Table 11 (model fit: $p<0.001$ ). Here, too, age, gender, preoperative pain, EHS classification, postoperative complications, ASA score, and presence of risk factors (in each case $p<0.001)$, as well as the use of a drain $(p=0.002)$, $\operatorname{BMI}(p=0.017)$, and EHS width classification $(p=0.035)$ had a significant relation to the rate of chronic pain requiring treatment (Fig. 2). Likewise, women were at notably higher risk of pain than men. The risk declined with increasing age and higher BMI. Preoperative pain, lateral, or combined EHS classification in comparison with medial, postoperative complications, higher ASA score, presence of at least one risk factor, and the use of drains were once again associated with a higher proportion of cases with chronic pain requiring treatment. Finally, higher width classification implied a 
Table 7 Multivariable analysis results for general complications, including odds ratio estimates with corresponding 95\% confidence intervals

\begin{tabular}{|c|c|c|c|c|c|c|}
\hline Parameter & $p$ value & Category & $p$ value (pairwise) & OR estimate & $95 \% \mathrm{CI}$ & \\
\hline \multirow[t]{3}{*}{ EHS width classification } & \multirow[t]{3}{*}{$<0.001$} & $\mathrm{~W} 3(>10 \mathrm{~cm})$ vs W1 $(<4 \mathrm{~cm})$ & $<0.001$ & 2.400 & 1.914 & 3.011 \\
\hline & & $\mathrm{W} 2(\geq 4-10 \mathrm{~cm})$ vs W1 $(<4 \mathrm{~cm})$ & $<0.001$ & 1.621 & 1.325 & 1.983 \\
\hline & & $\mathrm{W} 3(>10 \mathrm{~cm})$ vs W2 $(\geq 4-10 \mathrm{~cm})$ & $<0.001$ & 1.481 & 1.249 & 1.756 \\
\hline Risk factors & $<0.001$ & Yes vs no & & 1.500 & 1.288 & 1.747 \\
\hline Age [10-years-OR] & $<0.001$ & & & 1.169 & 1.095 & 1.248 \\
\hline \multirow[t]{3}{*}{ ASA score } & \multirow[t]{3}{*}{$<0.001$} & III/IV vs II & $<0.001$ & 1.431 & 1.221 & 1.677 \\
\hline & & III/IV vs I & 0.016 & 1.508 & 1.078 & 2.110 \\
\hline & & II vs I & 0.747 & 1.054 & 0.767 & 1.449 \\
\hline Drainage & $<0.001$ & Yes vs no & & 1.516 & 1.238 & 1.855 \\
\hline \multirow[t]{3}{*}{ EHS classification } & \multirow[t]{3}{*}{0.003} & Lateral vs medial & $<0.001$ & 0.679 & 0.544 & 0.849 \\
\hline & & Lateral vs combined & 0.012 & 0.670 & 0.490 & 0.914 \\
\hline & & Combined vs medial & 0.907 & 1.015 & 0.794 & 1.297 \\
\hline \multirow[t]{10}{*}{ Procedure } & \multirow[t]{10}{*}{0.175} & Open-IPOM vs laparoscopic_-IPOM & 0.023 & 1.344 & 1.042 & 1.733 \\
\hline & & Open-IPOM vs open—sublay & 0.049 & 1.229 & 1.001 & 1.508 \\
\hline & & Open-Onlay vs Open-IPOM & 0.151 & 0.776 & 0.550 & 1.097 \\
\hline & & Open-suture vs open-IPOM & 0.287 & 0.836 & 0.602 & 1.162 \\
\hline & & Open—sublay vs laparoscopic_-IPOM & 0.450 & 1.094 & 0.867 & 1.380 \\
\hline & & Open—suture vs laparoscopic-IPOM & 0.486 & 1.124 & 0.809 & 1.560 \\
\hline & & Open—suture vs open—onlay & 0.724 & 1.077 & 0.714 & 1.625 \\
\hline & & Open—onlay vs open—sublay & 0.770 & 0.954 & 0.696 & 1.307 \\
\hline & & Open—onlay vs laparoscopic_-IPOM & 0.817 & 1.044 & 0.727 & 1.498 \\
\hline & & Open—suture vs open—sublay & 0.863 & 1.027 & 0.755 & 1.397 \\
\hline Gender & 0.239 & Female vs male & & 1.092 & 0.943 & 1.264 \\
\hline \multirow[t]{3}{*}{ Preoperative pain } & \multirow[t]{3}{*}{0.394} & Yes vs no & 0.198 & 1.109 & 0.947 & 1.299 \\
\hline & & Unknown vs no & 0.371 & 1.132 & 0.863 & 1.485 \\
\hline & & Yes vs unknown & 0.878 & 0.980 & 0.756 & 1.270 \\
\hline BMI [5-points-OR] & 0.894 & & & 0.996 & 0.933 & 1.062 \\
\hline
\end{tabular}

higher risk of pain requiring treatment, but its significant difference was identified only on comparing W3 vs. W1. This corresponds to 80 out of 1000 patients with W3 suffering from pain requiring treatment compared to 67 out of 1000 patients with W1 width.

\section{Summary of results}

Figure 2 shows the main results from the aforementioned models in combination with the outcome of the bootstrap validation algorithm.

Higher EHS width classification (W2 vs. W1, W3 vs. W1) had a highly significantly unfavorable relation to the intraoperative and postoperative surgical complications, the complication-related reoperations, as well as to the general complications. On comparing W3 vs. W1, the former was additionally found to have a highly significantly unfavorable association with the complication-related reoperation rate, which in the case of $\mathrm{W} 2$ vs. W1 was only significantly unfavorable. Those model and bootstrap results thus demonstrate that EHS width classification has the highest negative association with the perioperative outcome in incisional hernia repair. Only the open vs. the laparoscopic technique likewise showed a highly significantly unfavorable relation to the postoperative surgical complication and the complication-related reoperation rates. Conversely, the open procedure compared with the laparoscopic technique had a highly significantly favorable association with the intraoperative complication rate. The only other significantly unfavorable relations identified were higher age to the intraoperative complications, higher ASA score to the general complications, risk factors to the postoperative surgical complications, complicationrelated reoperation rate, and the general complications. In contrast, lateral versus medial EHS classification had a significantly favorable association with the postoperative surgical complications, complication-related reoperation rate, and the general complications.

For the recurrence rate at 1-year follow-up, only higher EHS width classification, higher BMI, and lateral EHS classification were found to have a significantly unfavorable association, while the open vs. laparoscopic access 
Table 8 Multivariable analysis results for recurrence at 1-year follow-up, including odds ratio estimates with corresponding $95 \%$ confidence intervals

\begin{tabular}{|c|c|c|c|c|c|c|}
\hline Parameter & $p$ value & Category & $p$ value (pairwise) & OR estimate & $95 \% \mathrm{CI}$ & \\
\hline \multirow[t]{10}{*}{ Procedure } & \multirow[t]{10}{*}{$<0.001$} & Open—suture vs open—sublay & $<0.001$ & 2.928 & 2.389 & 3.590 \\
\hline & & Open—suture vs laparoscopic_-IPOM & $<0.001$ & 2.316 & 1.884 & 2.846 \\
\hline & & Open—suture vs open-IPOM & $<0.001$ & 2.005 & 1.603 & 2.509 \\
\hline & & Open—suture vs open—onlay & $<0.001$ & 2.006 & 1.500 & 2.682 \\
\hline & & Open-IPOM vs open—sublay & $<0.001$ & 1.460 & 1.207 & 1.766 \\
\hline & & Open—onlay vs open—sublay & 0.004 & 1.460 & 1.127 & 1.890 \\
\hline & & Open—sublay vs laparoscopic_-IPOM & 0.016 & 0.791 & 0.653 & 0.958 \\
\hline & & Open-IPOM vs laparoscopic-IPOM & 0.177 & 1.155 & 0.937 & 1.423 \\
\hline & & Open—onlay vs laparoscopic-IPOM & 0.324 & 1.154 & 0.868 & 1.535 \\
\hline & & Open—onlay vs open—IPOM & 0.998 & 1.000 & 0.752 & 1.329 \\
\hline \multirow[t]{3}{*}{ EHS width classification } & \multirow[t]{3}{*}{$<0.001$} & $\mathrm{~W} 2(\geq 4-10 \mathrm{~cm})$ vs W1 $(<4 \mathrm{~cm})$ & $<0.001$ & 1.417 & 1.213 & 1.656 \\
\hline & & $\mathrm{W} 3(>10 \mathrm{~cm})$ vs $\mathrm{W} 1(<4 \mathrm{~cm})$ & $<0.001$ & 1.548 & 1.270 & 1.886 \\
\hline & & $\mathrm{W} 3(>10 \mathrm{~cm})$ vs W2 $(\geq 4-10 \mathrm{~cm})$ & 0.305 & 1.092 & 0.923 & 1.293 \\
\hline \multirow[t]{3}{*}{ EHS classification } & \multirow[t]{3}{*}{$<0.001$} & Lateral vs medial & $<0.001$ & 1.366 & 1.174 & 1.590 \\
\hline & & Combined vs medial & 0.092 & 1.201 & 0.971 & 1.487 \\
\hline & & Lateral vs combined & 0.296 & 1.137 & 0.893 & 1.447 \\
\hline BMI [5-points-OR] & $<0.001$ & & & 1.101 & 1.044 & 1.161 \\
\hline Drainage & 0.073 & Yes vs no & & 1.155 & 0.987 & 1.352 \\
\hline Gender & 0.093 & Female vs male & & 0.899 & 0.794 & 1.018 \\
\hline Risk factors & 0.120 & Yes vs no & & 1.108 & 0.974 & 1.261 \\
\hline \multirow[t]{3}{*}{ Preoperative pain } & \multirow[t]{3}{*}{0.238} & Yes vs unknown & 0.098 & 0.836 & 0.677 & 1.034 \\
\hline & & Unknown vs no & 0.261 & 1.135 & 0.910 & 1.416 \\
\hline & & Yes vs no & 0.444 & 0.949 & 0.831 & 1.084 \\
\hline \multirow[t]{3}{*}{ ASA score } & \multirow[t]{3}{*}{0.279} & III/IV vs I & 0.148 & 1.200 & 0.937 & 1.537 \\
\hline & & III/IV vs II & 0.202 & 1.096 & 0.952 & 1.261 \\
\hline & & II vs I & 0.416 & 1.096 & 0.879 & 1.365 \\
\hline Age [10-years-OR] & 0.428 & & & 0.980 & 0.931 & 1.031 \\
\hline
\end{tabular}

technique showed a significantly favorable difference for the open approach.

The rates of pain on exertion, pain at rest, and chronic pain requiring treatment were highly significantly unfavorably associated with preoperative pain, female gender, and postoperative complications. Higher EHS width classification, risk factors, and lateral EHS classification had a significantly unfavorable association with the pain rates at 1-year follow-up. Higher age and higher BMI had a significantly favorable relation to pain rates.

\section{Subgroup of patients without follow-up}

In order to investigate whether there are relevant differences between the analysis population (limited to those patients with follow-up information $n=22,895)$ and the subgroup of patients without 1-year follow-up ( $n=13,637$, Fig. 1$)$, standardized differences were calculated for all patient- and operation-related variables, as well as post- and perioperative outcome variables. With the exception of age, with a mean difference of 2.4 years, for all other factors the standardized difference was found to be below 0.1. Thus, there is no bias in selection of patients due to the availability of follow-up information. The slightly higher age in the subgroup without follow-up demonstrates more difficulties in obtaining information related to outcome from older patients.

\section{Discussion}

The present multivariable analysis of 22,895 primary elective incisional hernia repairs from the Herniamed Registry investigated the potential influencing factors associated with outcome.

Multivariable models were estimated based on the confirmatory chosen, potential influencing parameters using all available data according to inclusion/exclusion criteria. The robustness of the results was assessed using a bootstrap algorithm per model with 1000 bootstrap samples each. 
Table 9 Multivariable analysis results for pain at rest at 1-year follow-up, including odds ratio estimates with corresponding $95 \%$ confidence intervals

\begin{tabular}{|c|c|c|c|c|c|c|}
\hline \multirow{2}{*}{$\frac{\text { Parameter }}{\text { Age [10-years-OR] }}$} & \multirow{2}{*}{$\frac{p \text { value }}{<0.001}$} & \multirow[t]{2}{*}{ Category } & \multirow[t]{2}{*}{$p$ value (pairwise) } & \multirow{2}{*}{$\begin{array}{l}\text { OR estimate } \\
0.799\end{array}$} & \multicolumn{2}{|l|}{$95 \% \mathrm{CI}$} \\
\hline & & & & & 0.771 & 0.827 \\
\hline \multirow[t]{3}{*}{ Preoperative pain } & \multirow[t]{3}{*}{$<0.001$} & Yes vs no & $<0.001$ & 1.680 & 1.513 & 1.865 \\
\hline & & Unknown vs no & $<0.001$ & 1.413 & 1.186 & 1.685 \\
\hline & & Yes vs unknown & 0.036 & 1.189 & 1.012 & 1.397 \\
\hline Gender & $<0.001$ & Female vs male & & 1.516 & 1.384 & 1.660 \\
\hline \multirow[t]{3}{*}{ EHS classification } & \multirow[t]{3}{*}{$<0.001$} & Lateral vs medial & $<0.001$ & 1.503 & 1.346 & 1.677 \\
\hline & & Lateral vs combined & 0.015 & 1.246 & 1.043 & 1.487 \\
\hline & & Combined vs medial & 0.020 & 1.206 & 1.030 & 1.412 \\
\hline Postoperative complication & $<0.001$ & Yes vs no & & 1.599 & 1.380 & 1.852 \\
\hline \multirow[t]{3}{*}{ ASA score } & \multirow[t]{3}{*}{$<0.001$} & III/IV vs I & $<0.001$ & 1.522 & 1.276 & 1.816 \\
\hline & & III/IV vs II & $<0.001$ & 1.209 & 1.090 & 1.342 \\
\hline & & II vs I & 0.003 & 1.259 & 1.079 & 1.469 \\
\hline BMI [5-points-OR] & 0.003 & & & 0.944 & 0.908 & 0.981 \\
\hline \multirow[t]{10}{*}{ Procedure } & \multirow[t]{10}{*}{0.027} & Open—suture vs laparoscopic-IPOM & 0.002 & 0.763 & 0.641 & 0.907 \\
\hline & & Open-suture vs open-sublay & 0.003 & 0.770 & 0.649 & 0.914 \\
\hline & & Open—suture vs open-IPOM & 0.020 & 0.797 & 0.659 & 0.965 \\
\hline & & Open—suture vs open—Onlay & 0.024 & 0.762 & 0.602 & 0.965 \\
\hline & & Open-IPOM vs laparoscopic_-IPOM & 0.575 & 0.957 & 0.820 & 1.117 \\
\hline & & Open-IPOM vs open—sublay & 0.632 & 0.966 & 0.840 & 1.112 \\
\hline & & Open—onlay vs open-IPOM & 0.684 & 1.046 & 0.842 & 1.299 \\
\hline & & Open—sublay vs laparoscopic_-IPOM & 0.882 & 0.990 & 0.866 & 1.131 \\
\hline & & Open—onlay vs open—sublay & 0.912 & 1.011 & 0.835 & 1.223 \\
\hline & & Open—onlay vs laparoscopic_-IPOM & 0.995 & 1.001 & 0.809 & 1.237 \\
\hline Drainage & 0.032 & Yes vs no & & 1.136 & 1.011 & 1.276 \\
\hline \multirow[t]{3}{*}{ EHS width classification } & \multirow[t]{3}{*}{0.036} & $\mathrm{~W} 3(>10 \mathrm{~cm})$ vs W1 $(<4 \mathrm{~cm})$ & 0.010 & 1.201 & 1.044 & 1.382 \\
\hline & & $\mathrm{W} 3(>10 \mathrm{~cm})$ vs W2 $(\geq 4-10 \mathrm{~cm})$ & 0.057 & 1.128 & 0.997 & 1.277 \\
\hline & & $\mathrm{W} 2(\geq 4-10 \mathrm{~cm})$ vs W1 $(<4 \mathrm{~cm})$ & 0.255 & 1.065 & 0.956 & 1.187 \\
\hline Risk factors & 0.365 & Yes vs no & & 1.045 & 0.950 & 1.149 \\
\hline
\end{tabular}

Higher EHS defect width classifications were found to have a highly significantly unfavorable relation to intraoperative complications, postoperative surgical complications as well as to general complications. Furthermore, defect width $>10 \mathrm{~cm}$ had a highly significantly unfavorable association with the complication-related reoperation rate. Defects of $\geq 4-10 \mathrm{~cm}$ were found to have only a significantly unfavorable relation to the complication-related reoperation rate. Only the open vs. the laparoscopic procedure was found to have, additionally, a highly significant unfavorable association with the postoperative surgical complications and the complication-related reoperation rate. Conversely, the open technique had a highly significantly favorable association with the intraoperative complication rate. Hence, the EHS width classification and surgical access route were identified as being the most important influencing factors for the perioperative outcome in incisional hernia repair. Higher patient age, the presence of risk factors, and higher BMI can also unfavorably influence the perioperative outcome.

In this analysis, no highly significant influencing factor was identified for recurrence at 1-year follow-up. Here, too, a larger defect width, higher BMI, and lateral EHS classification were seen to be significantly associated with recurrence.

The most important influencing factors for pain at rest, pain on exertion, and chronic pain requiring treatment at 1-year follow-up were preoperative pain and female gender. Other significantly unfavorable influencing factors were higher EHS width classification, presence of risk factors, higher ASA score, and lateral EHS classification.

As such, EHS width classification, open surgical technique, patient-reported preoperative pain, and female gender are the most important influencing factors for the outcome in incisional hernia repair. Accordingly, the findings presented here can be put to use for risk adjustment in incisional hernia repair. However that presupposes preoperative determination of the defect size by means of ultrasonography, computed 
Table 10 Multivariable analysis results for pain on exertion at 1-year follow-up, including odds ratio estimates with corresponding 95\% confidence intervals

\begin{tabular}{|c|c|c|c|c|c|c|}
\hline \multirow{2}{*}{$\frac{\text { Parameter }}{\text { Age [10-years-OR] }}$} & \multirow{2}{*}{$\frac{\mathrm{p} \text { value }}{<0.001}$} & \multirow[t]{2}{*}{ Category } & \multirow[t]{2}{*}{$p$ value (pairwise) } & \multirow{2}{*}{$\frac{\text { OR estimate }}{0.760}$} & \multicolumn{2}{|l|}{$95 \% \mathrm{CI}$} \\
\hline & & & & & 0.739 & 0.781 \\
\hline Gender & $<0.001$ & Female vs male & & 1.590 & 1.481 & 1.706 \\
\hline \multirow[t]{3}{*}{ Preoperative pain } & \multirow[t]{3}{*}{$<0.001$} & Yes vs no & $<0.001$ & 1.566 & 1.447 & 1.696 \\
\hline & & Unknown vs no & $<0.001$ & 1.378 & 1.204 & 1.576 \\
\hline & & Yes vs unknown & 0.045 & 1.137 & 1.003 & 1.289 \\
\hline \multirow[t]{3}{*}{ EHS classification } & \multirow[t]{3}{*}{$<0.001$} & Lateral vs medial & $<0.001$ & 1.510 & 1.383 & 1.648 \\
\hline & & Combined vs medial & $<0.001$ & 1.304 & 1.154 & 1.473 \\
\hline & & Lateral vs combined & 0.038 & 1.158 & 1.008 & 1.330 \\
\hline Postoperative complications & $<0.001$ & Yes vs no & & 1.421 & 1.258 & 1.606 \\
\hline \multirow[t]{3}{*}{ EHS width classification } & \multirow[t]{3}{*}{$<0.001$} & $\mathrm{~W} 3(>10 \mathrm{~cm})$ vs W1 $(<4 \mathrm{~cm})$ & $<0.001$ & 1.351 & 1.210 & 1.509 \\
\hline & & $\mathrm{W} 2(\geq 4-10 \mathrm{~cm})$ vs W1 $(<4 \mathrm{~cm})$ & $<0.001$ & 1.192 & 1.095 & 1.298 \\
\hline & & $\mathrm{W} 3(>10 \mathrm{~cm})$ vs W2 $(\geq 4-10 \mathrm{~cm})$ & 0.011 & 1.134 & 1.029 & 1.249 \\
\hline \multirow[t]{10}{*}{ Procedure } & \multirow[t]{10}{*}{$<0.001$} & Open-suture vs laparoscopic-IPOM & $<0.001$ & 0.726 & 0.635 & 0.831 \\
\hline & & Open—direkte naht vs open—sublay & 0.003 & 0.818 & 0.716 & 0.935 \\
\hline & & Open—suture vs open-IPOM & 0.005 & 0.808 & 0.696 & 0.937 \\
\hline & & Open—suture vs open—onlay & 0.009 & 0.782 & 0.649 & 0.941 \\
\hline & & Open—sublay vs laparoscopic_-IPOM & 0.025 & 0.888 & 0.800 & 0.985 \\
\hline & & Open-IPOM vs laparoscopic_-IPOM & 0.083 & 0.899 & 0.797 & 1.014 \\
\hline & & Open-Onlay vs Laparoscopic_-IPOM & 0.388 & 0.929 & 0.786 & 1.098 \\
\hline & & Open—onlay vs open—sublay & 0.555 & 1.046 & 0.900 & 1.217 \\
\hline & & Open-onlay vs open-IPOM & 0.706 & 1.033 & 0.871 & 1.225 \\
\hline & & Open-IPOM vs open—sublay & 0.822 & 1.013 & 0.907 & 1.130 \\
\hline Drainage & $<0.001$ & Yes vs no & & 1.189 & 1.086 & 1.303 \\
\hline BMI [5-points-OR] & 0.004 & & & 0.956 & 0.928 & 0.986 \\
\hline Risk factors & 0.004 & Yes vs no & & 1.115 & 1.035 & 1.200 \\
\hline \multirow[t]{3}{*}{ ASA score } & \multirow[t]{3}{*}{0.103} & III/IV vs I & 0.037 & 1.154 & 1.009 & 1.320 \\
\hline & & III/IV vs II & 0.142 & 1.064 & 0.980 & 1.155 \\
\hline & & II vs I & 0.168 & 1.084 & 0.966 & 1.217 \\
\hline
\end{tabular}

tomography, or magnetic resonance imaging [22-26]. Based on the radiologically measured defect width, the EHS width classification can then be used to estimate the expected outcomes.

Thus, this also serves as a good basis for the physician-patient consultation with regard to modification of risk factors, such as smoking and obesity, prior to surgery $[13,14]$. In particular, in the case of large defects, other risk factors should be reduced as far as possible [27]. Since incisional hernias become larger over time [28], with correspondingly poorer outcomes, watchful waiting should be carefully considered [29].

Based on the present analysis, patients with an incisional hernia who are at higher risk for perioperative complications and an unfavorable outcome at 1-year follow-up can be identified. Such patients should be operated on by experienced hernia surgeons. In particular, that applies to incisional hernia patients with a defect width of $>10 \mathrm{~cm}$ and who according to the guidelines [30, 31] should be operated on with an open technique. These patients have the highest perioperative complication risk, but are also susceptible to a significantly unfavorable influence on the recurrence rate and pain rates at 1-year follow-up. Independently of the defect width, female patients and patients with reported preoperative pain are at higher risk for the onset of chronic pain requiring treatment.

Missing or incorrect data limit a registry [20]. All responsible surgeons participating in the Herniamed Registry sign a contract for data correctness and completeness [20]. Missing data are indicated by the registry software [20]. Postoperative complications are once again reviewed at 1-year follow-up [20]. Experts can control data entry as part of the certification process of hernia centers [20]. The lack of follow-up in a relevant percentage (Fig. 1) of patients is another limitation of this registry study, but the subgroup analysis does not show any selection bias. The best safeguard is to compare the data with the literature 
Table 11 Multivariable analysis results for chronic pain requiring treatment at 1-year follow-up, including odds ratio estimates with corresponding $95 \%$ confidence intervals

\begin{tabular}{|c|c|c|c|c|c|c|}
\hline \multirow{2}{*}{$\begin{array}{l}\text { Parameter } \\
\text { Age [10-Jahres-OR] }\end{array}$} & \multirow{2}{*}{$\frac{p \text { value }}{<0.001}$} & \multirow[t]{2}{*}{ Category } & \multirow[t]{2}{*}{$p$ value (pairwise) } & \multirow{2}{*}{$\begin{array}{l}\text { OR estimate } \\
0.787\end{array}$} & \multicolumn{2}{|c|}{$95 \% \mathrm{CI}$} \\
\hline & & & & & 0.756 & 0.819 \\
\hline Gender & $<0.001$ & Female vs male & & 1.741 & 1.568 & 1.932 \\
\hline \multirow[t]{3}{*}{ Preoperative pain } & \multirow[t]{3}{*}{$<0.001$} & Yes vs no & $<0.001$ & 1.943 & 1.717 & 2.198 \\
\hline & & Unknown vs no & $<0.001$ & 1.529 & 1.246 & 1.877 \\
\hline & & Yes vs unknown & 0.011 & 1.271 & 1.056 & 1.529 \\
\hline \multirow[t]{3}{*}{ EHS classification } & \multirow[t]{3}{*}{$<0.001$} & Lateral vs medial & $<0.001$ & 1.564 & 1.382 & 1.769 \\
\hline & & Combined vs medial & $<0.001$ & 1.376 & 1.159 & 1.635 \\
\hline & & Lateral vs combined & 0.198 & 1.136 & 0.936 & 1.379 \\
\hline Postoperative complication & $<0.001$ & Yes vs no & & 1.570 & 1.331 & 1.851 \\
\hline \multirow[t]{3}{*}{ ASA score } & \multirow[t]{3}{*}{$<0.001$} & III/IV vs I & $<0.001$ & 1.804 & 1.465 & 2.221 \\
\hline & & II vs I & $<0.001$ & 1.448 & 1.204 & 1.741 \\
\hline & & III/IV vs II & $<0.001$ & 1.246 & 1.109 & 1.400 \\
\hline Risk factors & $<0.001$ & Yes vs no & & 1.220 & 1.097 & 1.356 \\
\hline Drainage & 0.002 & Yes vs no & & 1.230 & 1.079 & 1.403 \\
\hline BMI [5-points-OR] & 0.017 & & & 0.950 & 0.910 & 0.991 \\
\hline \multirow[t]{3}{*}{ EHS width classification } & \multirow[t]{3}{*}{0.035} & $\mathrm{~W} 3(>10 \mathrm{~cm})$ vs $\mathrm{W} 1(<4 \mathrm{~cm})$ & 0.012 & 1.228 & 1.047 & 1.439 \\
\hline & & $\mathrm{W} 2(\geq 4-10 \mathrm{~cm})$ vs W1 $(<4 \mathrm{~cm})$ & 0.067 & 1.122 & 0.992 & 1.270 \\
\hline & & W3 $(>10 \mathrm{~cm})$ vs W2 $(\geq 4-10 \mathrm{~cm})$ & 0.207 & 1.094 & 0.951 & 1.258 \\
\hline \multirow[t]{10}{*}{ Procedure } & \multirow[t]{10}{*}{0.102} & Open—suture vs open—IPOM & 0.032 & 0.794 & 0.643 & 0.981 \\
\hline & & Open—suture vs laparoscopic_-IPOM & 0.035 & 0.811 & 0.668 & 0.985 \\
\hline & & Open—IPOM vs open—sublay & 0.064 & 1.157 & 0.991 & 1.351 \\
\hline & & Open—sublay vs laparoscopic-IPOM & 0.107 & 0.883 & 0.759 & 1.027 \\
\hline & & Open—suture vs open-Onlay & 0.363 & 0.884 & 0.677 & 1.153 \\
\hline & & Open—suture vs open-Sublay & 0.389 & 0.919 & 0.759 & 1.113 \\
\hline & & Open—onlay vs open-IPOM & 0.390 & 0.899 & 0.705 & 1.146 \\
\hline & & Open—onlay vs laparoscopic-IPOM & 0.487 & 0.918 & 0.721 & 1.168 \\
\hline & & Open—onlay vs open—sublay & 0.724 & 1.040 & 0.837 & 1.293 \\
\hline & & Open—IPOM vs laparoscopic_-IPOM & 0.808 & 1.021 & 0.860 & 1.213 \\
\hline
\end{tabular}

[20]. The findings presented here are in concordance with the published data [12-15].

Furthermore, registry analyses do not allow for causal inference, but associations of variables can at least be detected when adjusting for known confounders and can thus be discussed.

In conclusion, this analysis of data from the Herniamed Registry demonstrates the very unfavorable association between high EHS width classification and intraoperative, postoperative and general complications, and complication-related reoperations, as well as its unfavorable relation to recurrences and pain rates at 1-year follow-up. Pain at rest and on exertion, as well as chronic pain requiring treatment is very unfavorably associated with female gender and preoperative pain and unfavorably related to lateral EHS classification and high ASA score. In comparison with the laparoscopic approach, the open sublay technique showed a highly, significantly reduced intraoperative complication rate, but highly increased postoperative complication and complication-related reoperation rates. Because incisional hernias become larger over time [28], with correspondingly poorer outcomes, watchful waiting should be carefully considered in incisional hernia repair. Patients with highly significant unfavorable factors should be treated by an experienced hernia surgeon.

\section{Compliance with ethical standards}

Conflict of interest Dr. Köckerling reports grants from Johnson \& Johnson, Norderstedt, grants from Karl Storz, Tuttlingen, grants from pfm medical, Cologne, grants from Dahlhausen, Cologne, grants from B Braun, Tuttlingen, grants from MenkeMed, Munich, grants from Bard, Karlsruhe, during the conduct of the study; personal fees from Bard, Karlsruhe, outside the submitted work. All other authors has nothing to disclose. 
Ethical approval As only cases of routine hernia surgery are documented in the Herniamed Registry, an ethical approval was not neccessary.

Human and animal rights This article does not contain any study with animals performed by any of the authors.

Informed consent All patients with routine hernia surgery documented in the Herniamed Registry have signed an informed consent declaration agreeing to participate.

Open Access This article is licensed under a Creative Commons Attribution 4.0 International License, which permits use, sharing, adaptation, distribution and reproduction in any medium or format, as long as you give appropriate credit to the original author(s) and the source, provide a link to the Creative Commons licence, and indicate if changes were made. The images or other third party material in this article are included in the article's Creative Commons licence, unless indicated otherwise in a credit line to the material. If material is not included in the article's Creative Commons licence and your intended use is not permitted by statutory regulation or exceeds the permitted use, you will need to obtain permission directly from the copyright holder. To view a copy of this licence, visit http://creativecommons.org/licenses/by/4.0/.

\section{References}

1. Kurian A, Gallagher S, Cheeyandira A, Josloff R (2010) Laparoscopic repair of primary versus incisional ventral hernias: time to recognize the differences? Hernia 14:383-387. https:// doi.org/10.1007/s10029-010-0649-0

2. Subramanian A, Clapp ML, Hicks SC, Awad SS, Liang MK (2013) Laparoscopic ventral hernia repair: primary versus secondary hernias. J Surg Res 181:e1-5. https://doi.org/10.1016/j. jss.2012.06.028

3. Stirler VMA, Schoenmaeckers EJP, de Haas RJ, Raymakers JTFJ, Rakic S (2014) Laparoscopic repair of primary and incisional ventral hernias: the differences must be acknowledged. Surg Endosc 28:891-895. https://doi.org/10.1007/s0046 4-013-3243-6

4. Köckerling F, Schug-Paß C, Adolf D, Reinpold W, Stechemesser B (2015) Is pooled data analysis of ventral and incisional hernia repair acceptable? Front Surg 2:15. https://doi.org/10.3389/ fsurg.2015.00015

5. Kroese LF, Gillion JF, Jeekel J, Kleinrensink GJ, Lange JF, The Hernia-Club Members (2018) Primary and incisional ventral hernias are different in terms of patient characteristics and postoperative complications - a prospective cohort study of 4565 patients. Int J Surg 51:114-119

6. Stabilini C, Cavallaro G, Dolce P, Giovannini SC, Corcione F, Frascio M, Sodo M, Merola G, Bracale U (2019) Pooled data analysis of primary ventral $(\mathrm{PVH})$ and incisional hernia $(\mathrm{IH})$ repair is no more acceptable: results of a systematic review and meta-analysis of current literature. Hernia 23:831-845. https:// doi.org/10.1007/s10029-019-02033-4

7. Holihan JL, Hanon C, Goodenough C, Flores-Gonzalez JR, Itani KM, Olavarria O et al (2017) Ventral hernia repair: a meta-analysis of randomized controlled trials. Surg Infect 18:647-658. https://doi.org/10.1089/sur.2017.029

8. Awaiz A, Rahman F, Hossain MB, Yunus RM, Khan S, Memon B, Memon MA (2015) Meta-analysis and systematic review of laparoscopic versus open mesh repair for elective incisional hernia. Hernia 19:449-463. https://doi.org/10.1007/s10029-015-1351-z
9. Jensen KK, Jorgensen LN (2015) Comment to: Meta-analysis and systematic review of laparoscopic versus open mesh repair for elective incisional hernia. Hernia 19:1025-1026. https://doi. org/10.1007/s10029-015-1412-3(Awaiz A et al. Hernia 2015; 19: 449-463)

10. Awaiz A, Rahman F, Hossain MB, Yunus RM, Khan S, Memon B, Memon MA, Jensen K, Jorgensen LN (2015) Reply to comment to Meta-analysis and systematic review of laparoscopic versus open mesh repair for elective incisional hernia. Hernia 19:1027-1029. https://doi.org/10.1007/s10029-015-1432-z

11. Al Chalabi H, Larkin J, Mehigan B, McCormick P (2015) A systematic review of laparoscopic versus open abdominal incisional hernia repair, with meta-analysis of randomized controlled trials. Int J Surg 20:65-74. https://doi.org/10.1016/j.ijsu.2015.05.050

12. Helgstrand F, Rosenberg J, Kehlet H, Jorgensen LN, Bisgaard T (2013) Nationwide prospective study of outcomes after elective incisional hernia repair. J Am Coll Surg 216:217-228. https:// doi.org/10.1016/j.jamcollsurg.2012.10.013

13. Owei L, Swendiman RA, Torres-Landa S, Dempsey DT, Dumon KR (2019) Impact of body mass index on minimally invasive ventral hernia repair: an ACS-NSQIP analysis. Hernia. https:// doi.org/10.1007/s10029-019-01944-6(epub ahead of print)

14. Kubasiak JC, Landin M, Schimpke S, Poirer J, Myers JA, Millikan KW, Luu MB (2016) The effect of tobacco use on outcomes of laparoscopic and open ventral hernia repairs: a review of the NSQIP dataset. Surg Endosc 31:2661-2666. https://doi. org/10.1007/s00464-016-5280-4

15. Kroese LF, Kleinrensink GJ, Lange JF, Gillion JF, the HerniaClub (2018) External Validation of the European Hernia Society Classification for Postoperative Complications after Incisional Hernia Repair: A Cohort Study of 2191 Patients. J Am Coll Surg. 226:223-229e1

16. Köckerling F, Hoffmann H, Adolf Weyhe D, Reinpold W, Koch A, Kirchhoff P (2019) Female gender as independent risk factor for chronic pain following primary elective incisional hernia repair-registry-based, propensity score-matched comparison of 22,895 patients. Hernia. https://doi.org/10.1007/s10029-01902089-2

17. Muysoms FE, Miserez M, Berrevoet F, Campanelli G, Champault GG, Chelala E, Dietz UA, Eker HH, El Nakadi I, Hauters P, Hidalgo Pascual M, Hoeferlin A, Klinge U, Montgomery A, Simmermacher RKF, Simons MP, Smietanski M, Sommeling C, Tollens T, Vierendeels T, Kingsnorth A (2009) Classification of primary and incisional abdominal wall hernias. Hernia 13:407-414. https://doi.org/10.1007/s10029-009-0518-x

18. Stechemesser B, Jacob DA, Schug-Pass C, Köckerling F (2012) Herniamed: an internet-based registry for outcome research in hernia surgery. Hernia 16:269-276. https://doi.org/10.1007/ s10029-012-0908.3

19. Kyle-Leinhase I, Köckerling F, Jørgensen LN, Montgomery A, Gillion JF, Rodrigues JAP et al (2018) Comparison of hernia registries: the CORE project. Hernia 22:561-575. https://doi. org/10.1007/s10029-017-1724-6

20. Köckerling F, Simon T, Hukauf M, Hellinger A, Fortelny R, Reinpold W, Bittner R (2018) The importance of registries in the postmarketing surveillance of surgical meshes. Ann Surg 268:1097-1104. https://doi.org/10.1097/SLA.0000000000 002326

21. Köckerling F, Simon T, Adolf D, Köckerling D, Mayer F, Reinpold W, Weyhe D (2018) Laparoscopic IPOM versus open sublay technique for elective incisional hernia repair: a registry-based, propensity score-matched comparison of 9907 patients. Surg Endosc. https://doi.org/10.1007/s00464-018-06629-2

22. Baucom RB, Ousley J, Feurer ID, Beveridge GB, Pierce RA, Holzman MD, Sharp KW, Poulose BK (2016) Patient reported outcomes after incisional hernia repair-establishing the ventral 
hernia recurrence inventory. Am J Surg 212:81-88. https://doi. org/10.1016/j.amjsurg.2015.06.007

23. Sanders DL, Kingsnorth A (2012) The modern management of incisional hernias. BMJ 344:e2843. https://doi.org/10.1136/bmj. e2843

24. Parikh KR, Al-Hawary M, Millet JD, Burney R, Finks J, Maturen K (2017) Incisional hernia repair: what the radiologist needs to know. AJR 209:1239-1246. https://doi.org/10.2214/ AJR.17.18137

25. Kroese LF, Sneiders D, Kleinrensink GJ, Muysoms F, Lange JF (2018) Comparing different modalities for the diagnosis of incisional hernia: a systematic review. Hernia 22:229-242. https://doi. org/10.1007/s10029-017-1725-5

26. Bellio G, Tommaso CM, Del Giudice R, Munegato G (2019) Preoperative abdominal computed tomography at rest and during valsalva's maneuver to evaluate incisional hernias. Surg Innov. https://doi.org/10.1177/1553350619849986(epub ahead of print)

27. Kaoutzanis C, Leichtle SW, Mouawad NJ, Welch KB, Lampman RM, Wahl WL, Cleary RK (2015) Risk factors for postoperative wound infections and prolonged hospitalization after ventral/incisional hernia repair. Hernia 19:113-123. https://doi.org/10.1007/ s10029-013-1155-y

28. Jensen KK, Arnesen RB, Christensen JK, Bisgaard T, Jørgensen LN (2019) Large incisional hernias increase in size. J Surg Res
244:160-165. https://doi.org/10.1016/j.jss.2019.06.016[Epub ahead of print]

29. Verhelst J, Timmermans L, van de Velde M, Jairam A, Vakalopoulos KA, Jeekel J, Lange JF (2015) Watchful waiting in incisional hernia: is it safe? Surgery 157:297-303. https://doi.org/10.1016/j. surg.2014.09.017

30. Bittner R, Bain K, Bansal VK, Berrevoet F, Bingener-Casey J, Chen D et al (2019) Update of guidelines for laparoscopic treatment of ventral and incisional abdominal wall hernias (International Endohernia Society (IEHS))-Part A. Surg Endosc 33:3069-3139. https://doi.org/10.1007/s00464-019-06907-7

31. Bittner R, Bain K, Bansal VK, Berrevoet F, Bingener-Casey J, Chen D et al (2019) Update of guidelines for laparoscopic treatment of ventral and incisional abdominal wall hernias (International Endohernia Society (IEHS)) - Part B. Surg. Endosc. https ://doi.org/10.1007/s00464-019-06908-6 [Epub ahead of print]

Publisher's Note Springer Nature remains neutral with regard to jurisdictional claims in published maps and institutional affiliations. 